\title{
High-power laser-plasma chemistry in planetary atmospheres
}

\author{
Svatopluk Civiš ${ }^{1}$ and Libor Juha ${ }^{2}$ \\ ${ }^{1}$ J. Heyrovský Institute of Physical Chemistry ASCR, Dolejškova 3, 18223 Prague 8, \\ Czech Republic \\ email: civis@jh-inst.cas.cz \\ ${ }^{2}$ Institute of Physics ASCR, Na Slovance 2, 18221 Prague 8,Czech Republic \\ email: juha@fzu.cz
}

\begin{abstract}
Large laser sparks created by a single shot of a high-power laser system were used for the laboratory simulation of the chemical consequences of high-energy-density events (lightning, high-velocity impact) in planetary atmospheres, e.g., the early Earth's atmosphere.
\end{abstract}

Keywords. Astrobiology, astrochemistry, plasmas, methods: laboratory, techniques: spectroscopic

It is currently a generally accepted opinion that Earth's early atmosphere was a mildly reduced mixture of molecular gases. This represents a crucial difficulty for the classical Miller's experiments. These experiments were carried out in a strongly reducing gaseous mixture. In a weakly reducing environment they give a poor yield of amino acids and other compounds of relevance to chemical evolution. In our study we intended to simulate impact shocks and lightning in the mixtures of molecular gases modeling Earth's early atmosphere with help of a focused beam from high-power laser systems (Civis et al. 2004, Babankova et al. 2006a and references cited therein). The main goal of this program is to diagnose the laser-produced plasma, determine its basic physical characteristics, and investigate their links to the chemical action of the laser spark in such a reaction system. Varying laser-plasma interaction conditions and composition of the gas mixtures of the chemical evolution during various stages of Earth's early atmosphere evolution was investigated in dependence on the kind and energetics of the initializing high-energy density event (i.e., impact shocks and atmospheric discharges).

A single laser pulse delivered from a Prague Asterix Laser System (a pulse duration of $400 \mathrm{ps}$ and a wavelength of $1.315 \mu \mathrm{m}$ ) with energy content $\leqslant 100 \mathrm{~J}$ was used for irradiation of the $\mathrm{CO}-\mathrm{N}_{2}-\mathrm{H}_{2} \mathrm{O}$ gas mixtures (mildly reducing systems) and their components at atmospheric pressure. The high pulse energy and the relatively short pulse duration result in the observed large volume of the laser spark.

Chemical consequences of the laser-produced plasma generation in such a gaseous system were investigated by gas/liquid chromatography and high-resolution Fourier transform infrared absorption spectrophotometry with a Bruker IFS 120 in the spectral interval from $500-7000 \mathrm{~cm}^{-1}$ at a resolution of $0.0035 \mathrm{~cm}^{-1}$. Several organic compounds were identified in the reaction mixture exposed to a few laser shots. The reaction mechanism of $\mathrm{CO}_{2}$ formation was investigated using stable isotope-labeled water $\mathrm{H}_{2}^{18} \mathrm{O}$ (Figure 1 ).

Optical emission spectra (OES) of the large laser spark were measured by a MS255 spectrometer (Oriel) equipped with time-resolved ICCD detector (Andor) in the spectral range of 350-1000 nm. A significant difference has been found in the optical spectra of LIDB plasmas created in $\mathrm{CO}$ and $\mathrm{N}_{2}$ containing mixtures in the static cell and gas puff (Babankova et al. 2006b). In the case of the jet, there is no examined emission from 


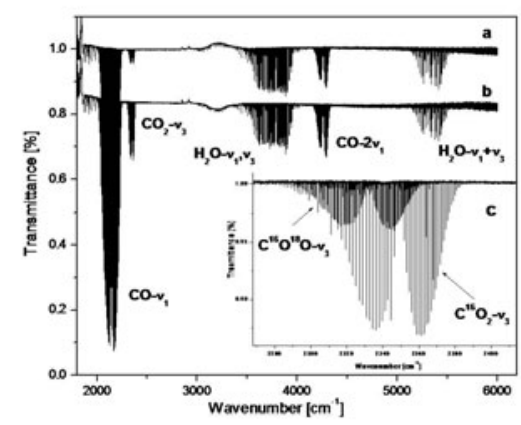

Figure 1. The high-resolution FTIR absorption spectra of (a) an unirradiated $\mathrm{CO}-\mathrm{N}_{2}-\mathrm{H}_{2} \mathrm{O}$ gaseous mixture, (b) the same mixture after ten laser sparks, and (c) the $\mathrm{CO}_{2}$ band composed of spectral components belonging to the $\mathrm{C}^{16} \mathrm{O}^{18} \mathrm{O}$ and $\mathrm{C}^{16} \mathrm{O}_{2}$ isotopomers.

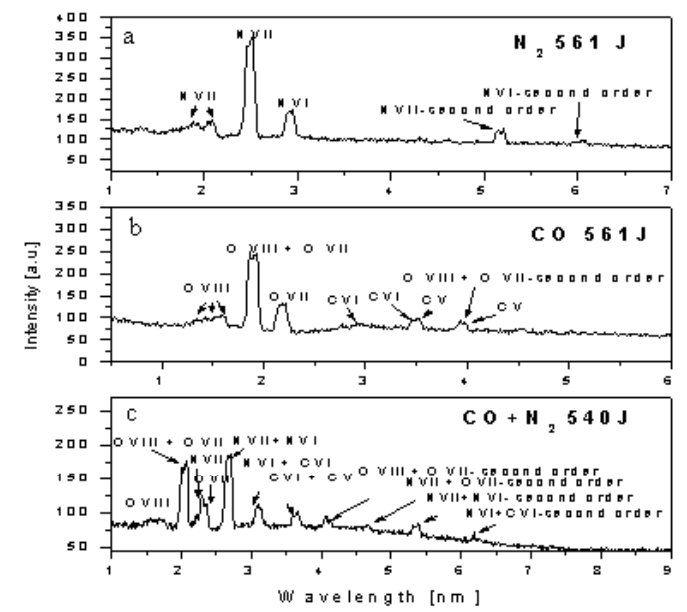

Figure 2. Soft x-ray emission spectra of laser sparks from pulse jet experiments: $\mathrm{N}_{2}$, (b) $\mathrm{CO}$ and (c) $\mathrm{CO}-\mathrm{N}_{2}$.

molecular species. It demonstrates that new molecules are formed in the phase of laser spark evolution where the LIDB plasma expands into the surrounding gas. Such a period does not take place in the gas puff because the LIDB plasma expands through the thin layer of helium directly into vacuum.

Experiments with the gas puff also provide the unique opportunity to look through the vacuum and observe the short-wavelength emission of LIDB plasma (Babankova et al. 2006b, Figure 2). This cannot be done in the static cell due to strong absorption of short-wavelength radiation in the cold, dense gas. It is expected that short-wavelength radiation could play a significant role in the chemical action of LIDB plasmas, initiating photochemical and/or radiation-chemical reactions in the surrounding gas.

This work was financially supported by Grant Agency of the Czech Republic (grant No. 203/06/1278) and the Czech Ministry of Education (grants LC510, LC528 and LA08024).

\section{References}

Civiš, S., Juha, L., Babankova, B., et al. 2004, Chem. Phys. Lett., 386, 169

Babankova, B., Civiš, S., \& Juha, L., 2006a, Prog. Quant. Electron., 30, 75

Babankova, B., Civiš, S., Juha, L., et al., 2006b, J. Phys. Chem., A110, 12113 